\title{
Umfassende Strategien zur Vermeidung sind gefragt
}

Innerhalb von 30 Tagen nach einem Krankenhausaufenthalt wegen Herzinsuffizienz, Herzinfarkt oder Pneumonie sind die Rehospitalisierungsraten in den USA hoch. Um geeignete Gegenmaßnahmen zu entwickeln, müssen zunächst die Gründe hierfür analysiert werden. K. Dharmarajan et al. haben dazu Daten aus dem Medicare-Versicherungssystem ausgewertet. JAMA 2013; 309: 355-363

Die Wissenschaftler zogen alle Daten der Versicherten heran, die zwischen 2007 und 2009 wegen einer der 3 Indikationen stationär behandelt worden waren und in diesem Zeitraum Anspruch auf den vollen Umfang der Versicherungsleistungen hatten. Zunächst ermittelten sie die Diagnosen und das zeitliche Auftreten der Rehospitalisierungen in den ersten 30 Tagen nach Entlassung und werteten sie dann sowohl für einzelne Tage, als auch für zeitliche Cluster (Tag 0-3, 0-7, $0-15$ und $0-30$ ) und aufeinanderfolgende Perioden (Tag 0-3, 4-7, 8-15 und 1630) nach erster Entlassung aus. Außerdem interessierte die Autoren, ob es Unterschiede der Rehospitalisierung je nach Patientencharakteristika gab, um gegebenenfalls vulnerable Gruppen zu identifizieren.

\section{Unspezifische Vulnerabilität $\nabla$}

Von 1330157 in den Jahren 2007-2009 wegen Herzinsuffizienz stationär behandelten Patienten mussten in den ersten 30 Tagen nach Entlassung 329308 wieder stationär aufgenommen werden $(24,8 \%)$. Bei den primär wegen eines akuten Myokardinfarkt behandelten 548834 Patienten waren es 108992 (19,9\%), bei den primär wegen einer Pneumonie stationär behandelten 1168624 Patienten 214239 (18,3\%). Die Diagnosen, die zur Wiederaufnahme führten, waren sehr unterschiedlich. Am häufigsten war dies zwar die eigentliche Diagnose, aber sie machte keineswegs den größten Anteil aus: Wegen derselben Diagnose rehospitalisiert wurden $35,2 \%$ der Patienten nach erster stationärer Behandlung einer

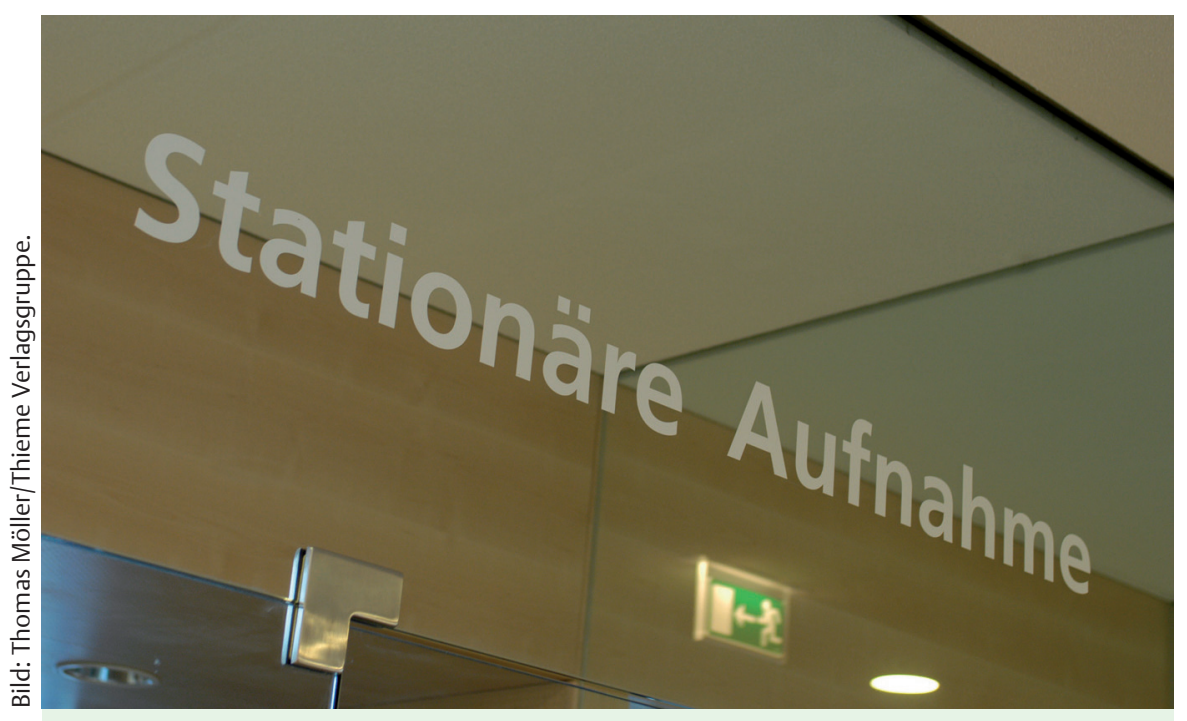

Laut Studie ist die stationäre Wiederaufnahme nach einem Herzinfarkt oder einer Pneumonie innerhalb eines Monats nach Entlassung häufig.
Herzinsuffizienz, 10\% nach einem akuten Myokardinfarkt und 22,4\% nach einer Indexpneumonie. Die ansonsten in allen 3 Kohorten herrschende Diagnosenvielfalt wurde in allen betrachteten Zeiträumen ähnlich beobachtet.

Die Zahl der stationären Aufnahmen war in den ersten 15 Tagen insgesamt etwas höher als danach (61\% nach Herzinsuffizienz, 67,6\% nach Herzinfarkt, 62,6\% nach Pneumonie). Rehospitalisierungen kamen aber über den ganzen 30-TageZeitraum hinweg häufig vor. Im Median erfolgte eine stationäre Wiederaufnahme bei den Herzinsuffizienz- und Pneumoniepatienten nach 12 Tagen, bei den Herzinfarktpatienten nach 10 Tagen. Weder die Diagnosen bei Rehospitalisierung noch der Zeitpunkt der stationären Wiederaufnahme zeigten eine Assoziation mit Alter, Geschlecht oder Herkunft.

\section{Posthospitalisierungssyndrom $\nabla$}

Die Autoren diskutieren ein allgemeines „Posthospitalisierungssyndrom“, eine generelle Vulnerabilität nach einem Krankenhausaufenthalt wegen einer der 3 Diagnosen, z.B. durch den Verlust an Kraft und Mobilität, entstandene Ernährungsdefizite, Schlafmangel oder neu auftretende Nebenwirkungen der im Krankenhaus eingeführten Medikamente. Um die hohen Rehospitalisierungsraten zu verringern, sind Strategien zu entwickeln, die sowohl Aspekte des Krankenhausaufenthalts selbst, als auch die Überleitung und eine umfassende Betreuung mindestens in den ersten $30 \mathrm{Ta}-$ gen nach Hospitalisierung betreffen.

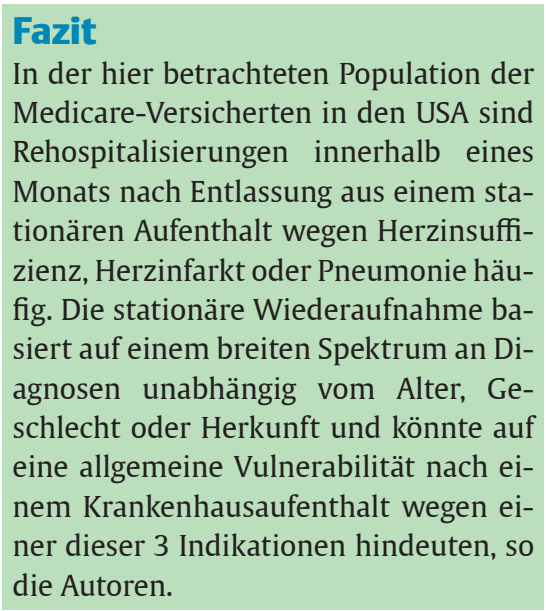

Friederike Klein, München 